\title{
The origin and spread of CRF85_BC, driven by heterosexual transmission among older people in Sichuan, China
}

\author{
Ling Su', Yi Feng ${ }^{2}$, Shu Liang ${ }^{1}$, Yali Zeng ${ }^{1}$, Yiping Li ${ }^{1}$, Hong Yang ${ }^{1}$, Li Ye ${ }^{1}$, Qiushi Wang ${ }^{1}$, Dongbin Wei ${ }^{1}$ Dan Yuan ,
} Wenhong Lai ${ }^{1}$ and Linglin Zhang ${ }^{1^{*}}$

\begin{abstract}
Background: CRF_BC recombinants, including CRF07_BC and CRF08_BC, were considered the predominant subtypes in China. Since the discovery of HIV-1 circulating recombinant form CRF 85_BC in Southwest China in 2016, this BC recombinant forms had been reported in different regions of China. However, the history and magnitude of CRF85_BC transmission were still to be investigated.

Method: We conducted the most recent molecular epidemiology of HIV-1 among newly reported HIV-1 infected patients in Sichuan in 2019 by sequencing and phylogenetic analysis of 1291 pol sequences. Then, we used maximum likelihood approach and the Bayesian Markov chain Monte Carlo (MCMC) sampling of pol sequences to reconstruct the phylogeographic and demographic dynamics of the CRF85_BC.

Results: HIV-1 CRF85_BC (68/1291, 5.27\%) became the fourth most prevalent strain revealing a significant increase in local population. CRF85_BC were only found in heterosexually infected individuals and the majority of CRF85_BC (95.45\%) were circulating among the people living with HIV aged 50 years and over (PLHIV50+), suggesting a unique prevalent pattern. The founder lineages of CRF85_BC were likely to have first emerged in Yunnan, a province of Southwest China bordering Sichuan, in the early 2000s. It then spread exponentially to various places (including Guangxi, Sichuan, et al) and became endemic around 2008.6 (2006.7-2010.2) in Sichuan.

Conclusion: Taken together, our findings on HIV-1 subtype CRF85_BC infections provided new insights into the spread of this virus and extended the understanding of the HIV epidemic in China.
\end{abstract}

Keywords: HIV-1, Recombinants, Heterosexually transmission

\section{Background}

The human immunodeficiency virus type 1 (HIV-1) epidemic remains a major public health burden in China. By the end of 2018, it is estimated that there are approximately 1.25 [1.10-1.40] million persons living with HIV/AIDS in China. During 2018, 81 [60-105] thousand new HIV infections occurred [1].

\footnotetext{
*Correspondence: zhangllsccdc@163.com

${ }^{1}$ Sichuan Provincial Center for Disease Control and Prevention, Center for AIDS/STD Control and Prevention, Chengdu 610041, Sichuan, China Full list of author information is available at the end of the article
}

In early 1990s, HIV-1 Thai subtypes $\mathrm{B}^{\prime}$ and $\mathrm{C}$ were co-circulated among injection drug users (IDUs) [2] in Yunnan province in southwestern China bordering the 'Golden Triangle', leading to active inter-subtype recombination which generated CRF07_BC, CRF08_BC, and a large number of unique recombinant forms $[3,4]$. In the initial period, drug trafficking activities were considered to be responsible for the rapid spread of CRF07_BC and CRF08_BC across China [5]. From 1978, as commercial sex activity increased across the country [6], the proportion of newly reported HIV infections/acquired immune

(c) The Author(s). 2020 Open Access This article is licensed under a Creative Commons Attribution 4.0 International License, which permits use, sharing, adaptation, distribution and reproduction in any medium or format, as long as you give appropriate credit to the original author(s) and the source, provide a link to the Creative Commons licence, and indicate if changes were made. The images or other third party material in this article are included in the article's Creative Commons licence, unless indicated otherwise in a credit line to the material. If material is not included in the article's Creative Commons licence and your intended use is not permitted by statutory regulation or exceeds the permitted use, you will need to obtain permission directly from the copyright holder. To view a copy of this licence, visit http://creativecommons.org/licenses/by/4.0/ The Creative Commons Public Domain Dedication waiver (http://creativecommons.org/publicdomain/zero/1.0/) applies to the data made available in this article, unless otherwise stated in a credit line to the data. 
deficiency syndrome (AIDS) patients through heterosexual transmission increased dramatically. Nowadays, CRF07_BC and CRF08_BC have become the most epidemic strains in China $[7,8]$ and drug-driven epidemic has been shifting to sexually transmission-driven epidemic [9]. There were other $\mathrm{B} / \mathrm{C}$ CRFs reported in the last few years, such as CRF57_BC (identified in Baoshan, Western Yunnan) [10], CRF62_BC and CRF64_BC (identified in Ruili/Dehong, Western Yunnan) [11]. However, there was scarce evidence that such $\mathrm{B} / \mathrm{C}$ recombinant forms had spread to other areas. In 2016, we reported a novel B/C recombinant form, CRF85_BC, among heterosexually infected patients in southern $\mathrm{Si}$ chuan (Yibin city) [12]. CRF85_BC was then reported in Anhui [13], Yunnan [14] and Northern Sichuan [15] (Guangyuan City). Due to lack of B or C epidemic history in the local high-risk population, the origin and the spread of CRF85_BC was still to be investigated.

Sichuan province is in the interior of Southwest China, adjacent to Yunnan and Guizhou in the South and Tibet in the West. Sichuan has a population of approximately 85 million people, and is a developing area in China. Since the initial HIV epidemic was fueled by IDU in 1990s, the HIV epidemic in Sichuan had become one of the worst in China. By 2018, there were 132, 680 HIV infections reported in Sichuan province, which ranked the first among all provinces/autonomous regions in China [16]. Several studies have focused on the HIV epidemic in IDUs $[17,18]$ and CRF07_BC $[19,20]$ in Liangshan prefecture in Sichuan, and reported that the infected IDUs in this region could serve as a source of transmission to other regions of China [20]. However, limited attention has been paid to the recent shift of the HIV-related risk behaviors from IDU to sexual contact, and also to other circulating HIV strains in this area.

Genetic and temporal dynamic analyses have been widely used to reconstruct the history of the HIV epidemic and have provided important information to aid in the development of strategies for the prevention of HIV-1 transmission. Here, we reported an investigation of the temporal and spatial dynamics of HIV CRF85_BC to give more clues about transmission of HIV strains in China.

\section{Methods}

\section{Study population}

A molecular epidemiological surveillance of Sichuan conducted among all newly HIV-1 diagnosed in April 2019. A total of 1573 antiretroviral-naive patient samples were collected at the first clinical visit. EDTA blood was collected with informed consent and $1291 \mathrm{HIV}-1$ pol sequences covering 1, 060 base pairs (HXB2: 2, 253-3, 312) were obtained as described previously [15]. Demographic data (age, gender \& self-identified ethnicity) and relevant information associated with HIV-1 transmission were abstracted from the national surveillance database. This study was reviewed and approved by the ethics committees of Sichuan Center for Disease Prevention and Control.

\section{HIV-1 subtyping and pairwise distance analysis}

HIV subtypes were assessed by phylogenetic analysis. The HIV-1 pol sequences were aligned with reference sequences of various subtypes and circulating recombinant forms (CRFs) from Los Alamos HIV sequence database (LANL, http://www.hiv.lanl.gov/). Multiple alignments were made automatically using the Mega version 7.0 [21] with minor manual adjustments. FastTree 2.3 was used to estimate an approximatelymaximum likelihood phylogenetic tree for $p o l$ sequences using the GTR + G+I nucleotide substitution model [22]. The phylogenetic tree's reliability was determined with local support values based on the ShimodairaHasegawa $(\mathrm{SH})$ test [23] and presented using FigTree v1.4.3 (http://tree.bio.ed.ac.uk/software/figtree/). For sequences of unknown HIV-1 subtype, the jumping profile hidden Markov model (jpHMM) (http://jphmm.gobics. de/submission_hiv) was applied to screen for recombination breakpoints.

Pairwise distances of CRF85_BC sequences collected from two cross-sectional surveys (in 2014 and 2019) were computed using the Tamura-Nei model in the MEGA v7.0 software [21] .

\section{CRF85_BC sequences dataset}

CRF85_BC HIV-1 pol sequences covering 1, 060 base pairs (HXB2: 2, 253-3, 312) were derived from multiple ways, form (i) molecular epidemiological surveillance of Sichuan in $2019(N=68)$, form (ii) molecular epidemiological surveillance of Sichuan in 2014 [24] $(N=14)$, from (iii) Drug resistance surveillance database in Sichuan CDC between 2012 and 2019 ( $N=58$ ), (iv) from other Molecular epidemiological investigation conducted in Sichuan [25] $(N=9)$, and (v) all other CRF85_BC (Yunnan: $n=2$; CQ: $n=1$ ) and the sequences (Yunnan: $n=7$; Anhui: $n=2$; Guangxi: $n=2$; HN: $n=1$ ) that are most closely related to each of the Sichuan CRF85_BC sequences with known sampling dates by BLAST search in the Los Alamos HIV database (LANL, http://www.hiv. lanl.gov/). All the sequences were confirmed as CRF85 $\mathrm{BC}$ by phylogenetic analysis mentioned above.

To avoid biases due to convergent evolution introduced by drug resistance selection rather than genealogical similarity, known surveillance drug resistance mutations (SDRM) have been excluded from the sequences prior to any analyses as described previously $[26,27]$. 


\section{Bayesian MCMC evolutionary analysis}

Estimation of evolutionary rate and the time of the most recent common ancestor (tMRCA) for CRF85_BC were performed by BEAST v1.8 [28]. A Bayesian skygrid model with Markov chain Monte Carlo (MCMC) inference under the relaxed lognormal molecular clock was selected as a reliable mode for this analysis. The MCMC chains were run 200 million times with 20,000 generations logged in, and the first $10 \%$ generations were discarded as burn-in. Bayesian MCMC output was analyzed using TRACER v1.5 [29], and all parameters were estimated from an ESS $>200$. The trees were summarized in a target tree using the Tree Annotator program and scanned using the Fig. Tree 1.4.3.

\section{Statistical analysis}

Chi-square test was used to compare the distributions of the population and demographic information among various HIV-1 strains. $P$ values less than 0.05 were considered as statistical significance. All statistical analyses were performed using SPSS v.20.0 software (IBM Company, New York, USA).

\section{Results}

Molecular epidemiological surveillance of Sichuan in 2019 Of the 1291 available sequences in the molecular epidemiological surveillance of Sichuan in 2019, CRF07_BC (676/1291, 52.36\%), CRF01_AE (341/1291, 26.41\%), CRF08_BC (114/1291, 8.83\%), and CRF85_BC (68/1291, $5.27 \%$ ) were the 4 most epidemic strains (Fig. 1a). CRF85_BC dominated the HIV-1 epidemics in Yibin (58/146), a southeast city in Sichuan Province, and was also detected in Luzhou (4/174), Leshan (3/120), Chengdu (2/312) and Mianyang (1/70) (Fig. 1b). Compare with the data of Sichuan molecular epidemiological surveillance in 2014 [24], the proportion of CRF85_BC in Sichuan increased from $3.66 \%(14 / 383)$ to $5.27 \%$, and it became more widely distributed. To further understand whether CRF85_BC was involved in ongoing transmission, we examined the sequence diversity by estimating pairwise distances between sequences in 2014 $(n=14)$ and sequences in 2019 $(n=68)$. We detected a much more diversity in 2019 (mean distance 0.023, SD 0.002) compared with 2014 (mean distance 0.010, SD 0.001), supporting an ongoing evolution of CRF85_BC overtime.

A

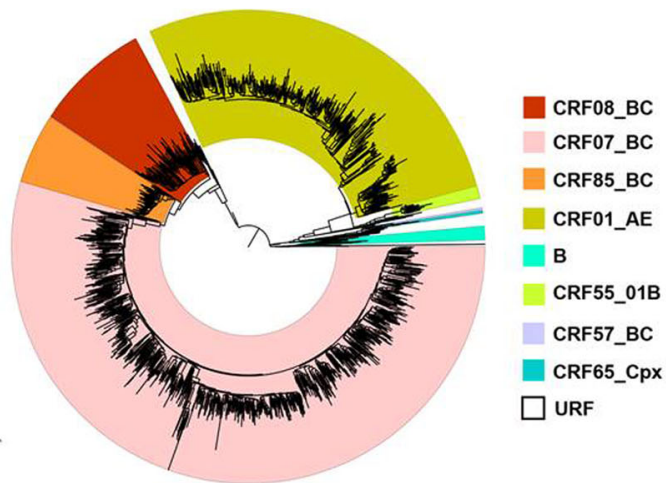

B

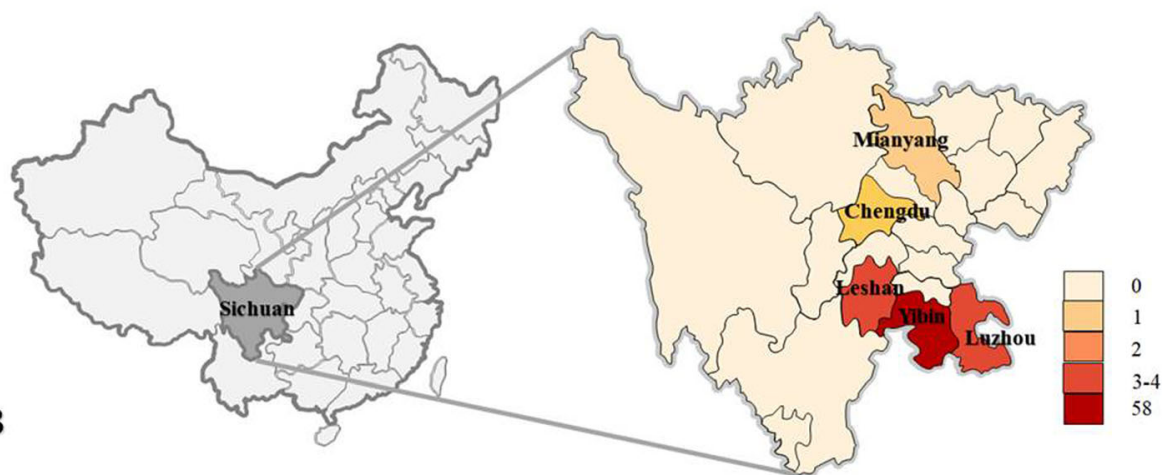

Fig. 1 The distribution of CRF85_BC from Sichuan in 2019. a Phylogenetic analysis of pol sequences from Sichuan in 2019. The phylogenetic tree was constructed using the approximately-maximum-likelihood method based on the pol region (HXB2: 2, 253 to 3, 312 nt) in FastTree 2.3. The nucleotide substitution mode was GTR $+\mathrm{G}+\mathrm{I}$. The subtypes and circulating recombinant forms are marked in different colors. The bootstrap value is indicated at relevant nodes. HIV-1 group $\mathrm{O}$ was chosen as an out-group in the rooted tree. The reference sequences from the Los Alamos HIV sequence database (http://hiv-web.lanl.gov/content/index). b The demographic distribution of CRF85_BC in Sichuan. Number of reported cases in different prefectures in Sichuan based on the molecular epidemiological surveillance of Sichuan in 2019 


\section{A heterosexual transmission of CRF85_BC in Sichuan Province}

The epidemiological and clinical features of the patients harboring CRF85_BC did not change significantly between 2014 and 2019 (Table 1). CRF85_BC were only found in heterosexually infected individuals, including commercial sexual transmission $(64.7 \%$ in 2019 and $42.9 \%$ in 2014), non-marital and non-commercial sexual transmission (30.9\% in 2019 and $21.4 \%$ in 2014) and spousal transmission (4.4\% in 2019 and $35.7 \%$ in 2014). The majority of CRF85_BC, in 2014(78.6\%) and 2019 (95.6\%), was circulating among the people living with HIV aged 50 years and over (PLHIV50+), significantly higher than other HIV CRFs $\left(P=0.000, x^{2}\right.$ test). Patients with low education levels took a relatively larger proportion.

\section{Estimated timeline and migration pathway of CRF85_BC}

To elucidate the sources and dissemination routes of endemic CRF85_BC geographically, we constructed maximum clade credibility (MCC) tree based on 149 Sichuan sequences and 15 sequences from other provinces in China using Bayesian geographical evolution

Table 1 Characteristics of patients harboring CRF85_BC in the molecular epidemiological surveillance of Sichuan conducted in 2014 and 2019

\begin{tabular}{|c|c|c|}
\hline Characteristics & 2014 & 2019 \\
\hline \multicolumn{3}{|l|}{ Region[\%; (n)] } \\
\hline Yibin & $92.9(13)$ & $85.3(58)$ \\
\hline Panzhihua & $7.1(1)$ & - \\
\hline Luzhou & - & $5.9(4)$ \\
\hline Chengdu & - & $2.9(2)$ \\
\hline Leshan & - & $4.4(3)$ \\
\hline Mianyang & - & $1.5(1)$ \\
\hline Gender & - & \\
\hline Male & $57.1(8)$ & $73.5(50)$ \\
\hline Female & $42.9(6)$ & $26.5(18)$ \\
\hline \multicolumn{3}{|l|}{ Age at diagnosis (year) } \\
\hline $30-49$ & $21.4(3)$ & $4.4(3)$ \\
\hline $50-60$ & $35.7(5)$ & $19.1(13)$ \\
\hline$\geq 60$ & $42.9(6)$ & $76.5(52)$ \\
\hline \multicolumn{3}{|l|}{ Modality of transmission } \\
\hline Commercial Heteosexual & $42.9(6)$ & $64.7(44)$ \\
\hline Non-marital and non-commercial Heteosexual & $21.4(3)$ & $30.9(21)$ \\
\hline Spousal Heteosexual & $35.7(5)$ & $4.4(3)$ \\
\hline \multicolumn{3}{|l|}{ Education level } \\
\hline Primary school and below & $78.6(11)$ & $75.0(51)$ \\
\hline Middle school & $21.4(3)$ & $23.5(16)$ \\
\hline High school & - & $1.5(1)$ \\
\hline
\end{tabular}

method. The corresponding median coefficient of rate variation was $0.33(95 \%$ highest posterior density (HPD): 0.18-0.49), supporting the selection of a relaxed molecular clock model. Both Bayesian skyline plot and Bayesian skygrid analysis showed that HIV-1 CRF85_BC experienced 2 fast growth phases during 2011-2014 and 2017-2019 (Fig. 2a) with a mean growth rate of $2.5^{*} 10^{-3}$ per year [95\% HPD: $2.0^{*} 10^{-3}-3.0^{*} 10^{-3}$ ]. The tMRCA was inferred to be 2005.4 (95\% HPD: 2002.3-2007.9), implying the origin time of the CRF85_BC (Table 2).

The MCC tree showed that CRF85_BC from Yunnan were located at the root of the tree (posterior probability $(\mathrm{PP}=1)$. Sequentially, virus spread form Yunnan to Sichuan $(\mathrm{PP}=0.9911)$, Guangxi $(\mathrm{PP}=0.9978)$, and other regions. The first place of the viral introduction into $\mathrm{Si}$ chuan was probably Yibin in 2008.6 (2006.7-2010.2). An explosive outbreak was then found in the heterosexual elderly people in this city. Several small clusters further suggested that the virus became epidemic in many cities of Sichuan (Luzhou, Guangyuan, et al.) and had rapidly spread to other provinces (such as Liaoning, Anhui and Chongqing) (Fig. 2b).

Notably, most Sichuan sequences originated from Yibin, except for 4 sequences. A Guangyuan and Mianyang sequence directly formed a sub-cluster with a sequence from Yunnan $(\mathrm{PP}=0.9965)$ while a Luzhou strain grouped significantly $(\mathrm{PP}=0.9977)$ with the sequences from Guangxi (Fig. 2b).

\section{Discussion}

Consistent with the differences in viral diversity among geographical regions in China [8], over $66.5 \%$ circulating HIV strains in Sichuan were defined as CRF_BC including CRF07_BC, CRF08_BC and CRF85_BC et al. Hence, CRF_BC infections became a more serious public health issue, which necessitated a deeper understanding of the CRF_BC epidemic patterns in China. CRF85_BC, a newly identified CRF_BC, were the fourth most prevalent HIV strains $(68 / 1291,5.27 \%)$ in Sichuan and was dominating the HIV-1 epidemic in a southwestern city, i e. Yibin city.

In the present study, we only found CRF85_BC prevalent in heterosexually infected individuals. In molecular epidemiological surveillance of Sichuan in 2019, 64.7\% of CRF85_BC were transmission via commercial sex. Other early studies about CRF07_BC and CRF08_BC suggested that CRF_BC had spread from IDU to the general population by sexual transmission [30]. Due to lack of the early CRF85_BC sequence, we could not rule out the possibility that this kind of CRF_BC originated from other high-risk groups such as IDUs or former plasma donors (FPDs) [13]. However, commercial sex transmission was at least an important driving factor of CRF85_BC becoming endemic in some local areas. 


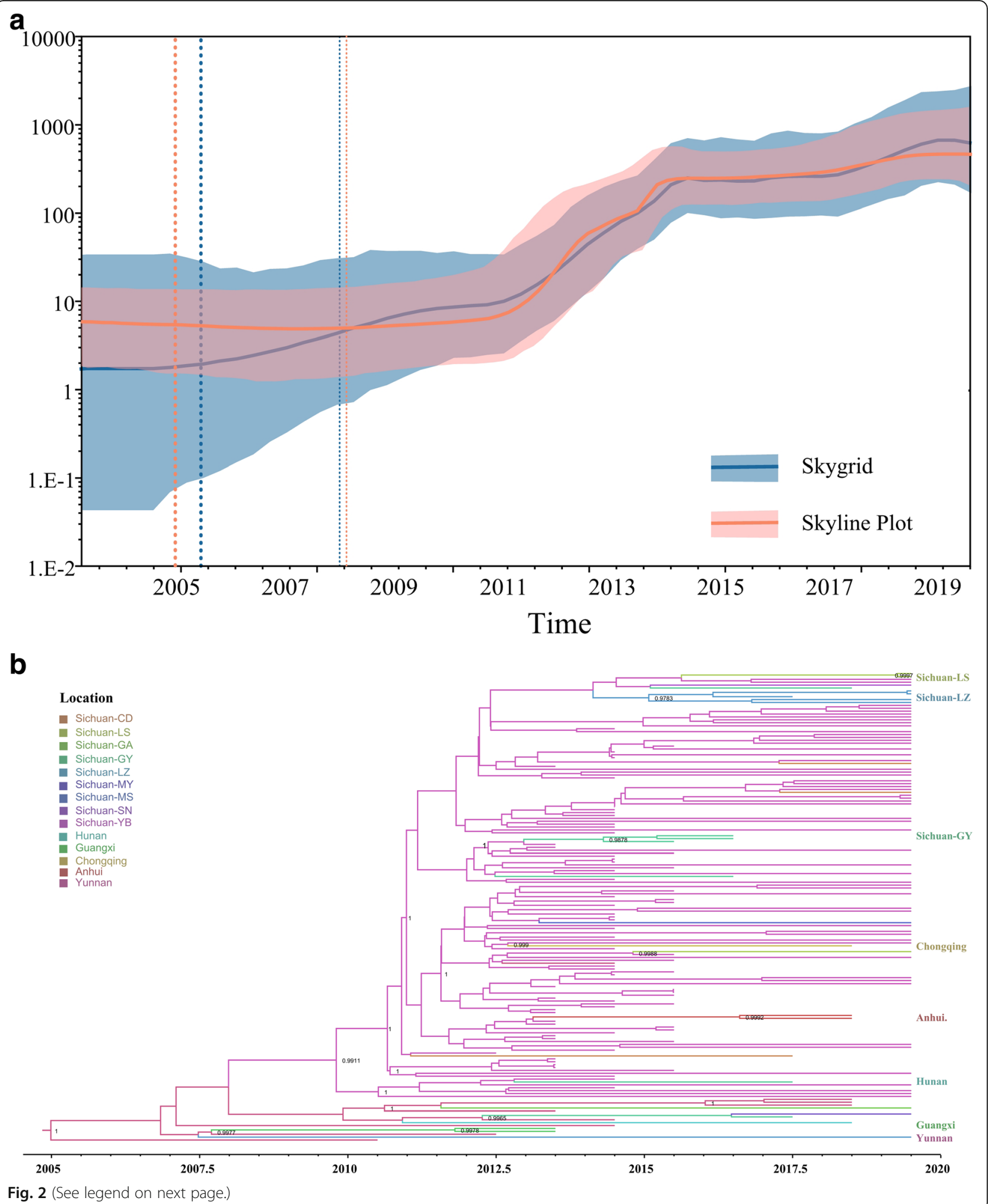


(See figure on previous page.)

Fig. 2 Prevalence and demographic history of CRF85_BC in Sichuan. a The Bayesian skyline plot and Bayesian skygrid analysis of CRF85_BC. The Bayesian skyline plot and Bayesian skygrid analysis estimated the past population dynamics of CRF85_BC. The thick solid line in the plot represents the median estimate, and the shaded region represents the 95\% HPD credible region. $\mathbf{b}$ The MCC tree of CRFF85_BC. The MCC tree was constructed based a data set of 148 CRF85_BC pol region (HXB2: 2, 253 to 3, 312 nt) using Bayesian MCMC analysis implemented in BEAST v 1.8.4. The analysis was performed using an uncorrelated lognormal relaxed clock model in GTR $+1+G 4$ nucleotide substitution model under an exponential coalescent model. The MCMC analysis was run for 200 million generations, with sampling every 20,000 generations. The different color branches indicate the strains from different areas, respectively. Sichuan-CD (Chengdu), Sichuan-LS (Leshan), Sichuan-GA (Guangan), SichuanGY (Guangyuan), Sichuan-LZ (Luzhou), Sichuan-MY (Mianyang), Sichuan-MS (Meishan), Sichuan-SN (Suining), Sichuan-YB (Yibin)

Notably, the majority of cases infected by CRF85_BC were PLHIV50+ cases, most of whom were loweducated. PLHIV50+ have been significantly increasing in the past two decades globally [31]. The proportion of PLHIV50+ around the world increased substantially from $8 \%$ in 2000 to $16 \%$ in 2016 and is expected to reach an estimated $21 \%$ by 2020 [32]. Poor awareness about safer sex practices and underestimating their risk of contracting HIV made them extremely vulnerable to HIV.

Analysis of 148 PR/RT sequences from CRF85_BC infected individuals sampled between 2011 and 2019 revealed that this strain had not just become endemic in Sichuan but also spread to some other provinces in China. Until now, at least 6 provinces have been affected and had a most recent common ancestor (tMRCA) is estimated at around 2005. Yunnan is considered to be the origin area. Previous studies in Yunnan between 2009 and 2012 revealed several CRF_BC HIV-1 strains circulating among local sexually active youth, which is coincident with the gateway position of Yunnan in China's HIV epidemic history [33]. By Bayesian skyline plot and Bayesian skygrid analysis, the exponential increase in the effective number of CRF85_BC indicated that the virus experienced 2 fast growth phases during 2011-2014 and 2017-2019.

CRF85_BC dispersion showed a strong geographic compartmentalization. After its origin in Yunnan, CRF85_BC almost at the same time spread to neighboring provinces such as Sichuan around 2008.6 (2006.7-

Table 2 Estimated dates of CRF85_BC origin in different areas

\begin{tabular}{llll}
\hline Regions & & Year & 95\%HPD \\
\cline { 1 - 2 } Province & City & & \\
\hline Yunnan & & 2005.4 & $2002.3-2007.9$ \\
Guangxi & & 2012.3 & $2011.3-2013.2$ \\
Anhui & & 2017.9 & $2016.8-2018.5$ \\
Sichuan & & \\
& Yibin & 2008.6 & $2006.7-2010.2$ \\
& Luzhou & 2014.3 & $2012.7-2015.8$ \\
& Leshan & 2019.1 & $2018.2-2019.5$ \\
& Guangyuan & 2013.6 & $2012.3-2015.0$ \\
\hline
\end{tabular}

2010.2), Guangxi around 2012.3 (2011.3-2013.2). The first place where the virus was introduced into Sichuan was probably Yibin city, which was located in the junction of Yunnan, Guizhou and Sichuan Provinces. In recent years, the local epidemic situation had deteriorated rapidly [34]. The explosive epidemic of CRF85_BC was found in the heterosexual elderly people in this city, and $39.7 \%$ (58/146) HIV cases locally were infected by CRF85_BC. Phylogeographical reconstruction further suggested that CRF85_BC spread from Yibin to other cities of Sichuan and even other provinces. This virus was identified in 9 cities in Sichuan, 3 of which probably had an endemic, such Luzhou, Leshan and Guangyuan. Leshan and Luzhou were both bordering with Yibin. Guangyuan is a northeastern city in Sichuan. According to another research, CRF85_BC infected people with Yibin household registration travelled to and resided in Guangyuan, further adding fuel to local endemic.

CRF85_BC entered Sichuan probably through multiple introduction events. In phylogenetic trees, some single strains of Sichuan also formed sub-clusters with Yunnan or Guangxi sequences. However, not all imported cases of Sichuan caused local epidemics.

\section{Conclusions}

Our study is the first report to clarify the phylogeographic and demographic dynamics of CRF 85_BC, a newly-identified and increasingly prevalent HIV-1 strain, in Sichuan, China. This study also highlights that heterosexual behavior among older people played an important role in the contribution of CRF 85_BC transmission. The origin of CRF 85_BC was traced back to Yunnan around 2005, spread exponentially to various places (including Guangxi, Sichuan, et al), and later on became endemic around 2008.5 (2007.0-2010.5) in Sichuan. Our findings provide novel insights into the dissemination of a novel HIV-1 virus and extend the understanding of the HIV epidemic in China.

\footnotetext{
Abbreviations

PLHIV50 +: The people living with HIV aged 50 years and over; CRFs: Circulating recombinant forms; IDUs: Injecting drug users; LANL: LOS Alamos National Laboratory; MCMC: Bayesian Markov chain Monte Carlo; SDRM: Surveillance drug resistance mutations (SDRM); SH: ShimodairaHasegawa; tMRCA: The most recent common ancestor; HPD: Highest posterior density; PP: Posterior probability
} 


\section{Acknowledgments}

The authors would like to thank staffs in Division of Virology and Immunology, National Center for AIDS/STD Control and Prevention (NCAIDS), China CDC for technical assistance. The opinions expressed herein show the collective views of the coauthors and do not necessarily represent the official position of the Sichuan Provincial Center for Disease Control and Prevention, Center for AIDS/STD Control and Prevention.

\section{Authors' contributions}

LS and LZ designed the study; YL1 (corresponding to Yiping Li), HY, YL2 (corresponding to Ye Li) YL, HY, LY jk and DW participated in the process of sequences editing and phylogenetic analyses; YF, LS and YZ performed statistical analysis; SL, DY and WL collected sample and the demographic data; LS, QW and YF participated in the writing process. All authors read and approved the final manuscript.

\section{Funding}

This study was supported by the Sichuan Science and Technology Project (2017JY0302). The funders had no role in study design, data collection and analysis, decision to publish, or preparation of the manuscript.

\section{Availability of data and materials}

The CRF85_BC sequences determined in this study are available in GenBank under Accession Numbers MT522696 - MT522844. Other datasets used and/ or analyzed during the current study available from the corresponding author on reasonable request.

\section{Ethics approval and consent to participate}

This study was reviewed and approved by the ethics committees of Sichuan Center for Disease Prevention and Control. All participants signed written informed consents prior to sample donations.

\section{Consent for publication}

Not applicable.

\section{Competing interests}

The authors declare no competing financial interests exist.

\section{Author details}

${ }^{1}$ Sichuan Provincial Center for Disease Control and Prevention, Center for AIDS/STD Control and Prevention, Chengdu 610041, Sichuan, China. ${ }^{2}$ Division of Virology and Immunology, National Center for AIDS/STD Control and Prevention (NCAIDS), China CDC, Beijing, China.

Received: 12 February 2020 Accepted: 8 October 2020

\section{Published online: 19 October 2020}

\section{References}

1. Lyu P, Chen FF. National HIV/AIDS epidemic estimation and interpretation in China. Zhonghua liu xing bing xue za zhi = Zhonghua liuxingbingxue zazhi. 2019;40(10):1191-6.

2. Feng $Y$, Takebe $Y$, Wei H, He X, Hsi JH, Li Z, Xing H, Ruan Y, Yang Y, Li F, et al. Geographic origin and evolutionary history of China's two predominant HIV-1 circulating recombinant forms, CRF07_BC and CRF08_ BC. Sci Rep. 2016;6:19279.

3. Yang R, Xia X, Kusagawa S, Zhang C, Ben K, Takebe Y. On-going generation of multiple forms of HIV-1 intersubtype recombinants in the Yunnan Province of China. AIDS. 2002;16(10):1401-7.

4. Nikolopoulos GK, Kostaki EG, Paraskevis D. Overview of HIV molecular epidemiology among people who inject drugs in Europe and Asia. Infect Genet Evol. 2016;46:256-68

5. Xiao Y, Kristensen S, Sun J, Lu L, Vermund SH. Expansion of HIV/AIDS in China: lessons from Yunnan Province. Soc Sci Med. 2007;64(3):665-75.

6. Zhang L, Chow EP, Jing J, Zhuang X, Li X, He M, Sun H, Gorgens M, Wilson $D$, Wang $L$, et al. HIV prevalence in China: integration of surveillance data and a systematic review. Lancet Infect Dis. 2013;13(11):955-63.

7. Xiao P, Li J, Fu G, Zhou Y, Huan X, Yang H. Geographic distribution and temporal trends of HIV-1 subtypes through heterosexual transmission in China: a systematic review and meta-analysis. Int J Environ Res Public Health. 2017;14(7):830.
8. He X, Xing H, Ruan Y, Hong K, Cheng C, Hu Y, Xin R, Wei J, Feng Y, Hsi JH, et al. A comprehensive mapping of HIV-1 genotypes in various risk groups and regions across China based on a nationwide molecular epidemiologic survey. PLoS One. 2012;7(10):e47289.

9. Chen H, Wu X, Chen L, Lu H, Tang Z, Shen Z, Pan SW, Ruan Y, Shao Y. Rapidly spreading human immunodeficiency virus epidemic among older males and associated factors: a large-scale prospective cohort study in rural Southwest China. Sex Transm Dis. 2019;46(4):234-9.

10. Wei H, Liu Y, Feng Y, Hsi J, Xing H, He X, Liao L, Yutaka T, Li J, Shao Y. Genome sequence of a novel HIV-1 circulating recombinant form (CRF57_ BC) identified from Yunnan, China. AIDS Res Hum Retrovir. 2014;30(4):384-8.

11. Hsi J, Wei $H$, Xing $H$, Feng $Y$, He $X$, Liao L, Jia M, Wang N, Ning C, Shao $Y$. Genome sequence of a novel HIV-1 circulating recombinant form (CRF64_BC) identified from Yunnan, China. AIDS Res Hum Retrovir. 2014;30(4):389-93.

12. Su L, Wei D, Yang H, Zeng Y, Hu Y, Yuan D, Feng L, Ruan Y, Qin G, Liang S. Identification of a novel HIV-1 circulating recombinant form (CRF85_BC) in Sichuan, China. AIDS Res Hum Retrovir. 2016;32(9):895-9.

13. Wu J, Zhang $Y$, Shen $Y$, Wang $X$, Xing H, Yang $X$, Ding $X, H u B$, Li H, Han J, et al. Phylogenetic analysis highlights the role of older people in the transmission of HIV-1 in Fuyang, Anhui Province, China. BMC Infect Dis. 2019;19(1):562.

14. Chen M, Ma Y, Chen H, Dai J, Luo H, Yang C, Dong L, Jin X, Yang M, Yang L, et al. Spatial clusters of HIV-1 genotypes in a recently infected population in Yunnan, China. BMC Infect Dis. 2019;19(1):669.

15. Su L, Liang S, Hou X, Zhong P, Wei D, Fu Y, Ye L, Xiong L, Zeng Y, Hu Y, et al. Impact of worker emigration on HIV epidemics in labour export areas: a molecular epidemiology investigation in Guangyuan, China. Sci Rep. 2018; 8(1): 16046.

16. NCSTD N. National AIDS venereal disease in December 2017; 2018. p. 02.

17. Yang $Y$, Latkin CA, Luan $R$, Yang C. A cross-sectional study of the feasibility of pharmacy-delivered harm reduction services among people who inject drugs in Xichang, China. BMC Public Health. 2015:15:885.

18. Yang Y, Latkin C, Luan R, Yang C. Reality and feasibility for pharmacydelivered services for people who inject drugs in Xichang, China: comparisons between pharmacy staff and people who inject drugs. Int J Drug Policy. 2016;27:113-20.

19. Li L, Wei D, Hsu WL, Li T, Gui T, Wood C, Liu Y, Li H, Bao Z, Liu S, et al. CRF07 BC strain dominates the HIV-1 epidemic in injection drug users in Liangshan prefecture of Sichuan, China. AIDS Res Hum Retrovir. 2015;31(5): 479-87.

20. Meng Z, Xin R, Abubakar YF, Sun J, Wu H, Lu J, Ni Y, Zhang X, Xu J. Five new CRF07_BC near full-length sequences isolated from Sichuan, China. AIDS Res Hum Retrovir. 2013;29(1):191-7.

21. Kumar S, Stecher G, Tamura K. MEGA7: molecular evolutionary genetics analysis version 7.0 for bigger datasets. Mol Biol Evol. 2016;33(7):1870-4.

22. Price MN, Dehal PS, Arkin AP. FastTree 2 - approximately maximumlikelihood trees for large alignments. PLoS One. 2010;5(3):e9490.

23. Liu K, Linder CR, Warnow T. RAxML and FastTree: comparing two methods for large-scale maximum likelihood phylogeny estimation. PLoS One. 2011; 6(11):e27731.

24. Ling $S$, Hong Y, Ying H, Li Y, Dan Y, Wei D, Zhou G, Jia Y, Liu H, Qin G. Molecular epidemiological study on HIV-1 in Sichuan province in 2014. Chin J Aids Std. 2016;22(12):946-9.

25. Ling S, Li Y, Yang L, Jiafeng Z, Shundong W, Shuibin L, Xueqin H, Dan Y, Hong Y, Dongbing W. HIV-1 molecular epidemiological survey of people over 50 years old in Sichuan. Chinese J Aids Std. 2018;24(8):788-92.

26. Bennett DE, Camacho RJ, Otelea D, Kuritzkes DR, Fleury H, Kiuchi M, Heneine W, Kantor R, Jordan MR, Schapiro JM, et al. Drug resistance mutations for surveillance of transmitted HIV-1 drug-resistance: 2009 update. PLoS One. 2009;4(3):e4724

27. Paraskevis D, Beloukas A, Stasinos K, Pantazis N, de Mendoza C, Bannert N, Meyer L, Zangerle R, Gill J, Prins M, et al. HIV-1 molecular transmission clusters in nine European countries and Canada: association with demographic and clinical factors. BMC Med. 2019;17(1):4.

28. Drummond AJ, Rambaut A. BEAST: Bayesian evolutionary analysis by sampling trees. BMC Evol Biol. 2007;7:214.

29. Rambaut A SM, Xie D, Drummond AJ.: Tracer v1.6. . http://beast.bio.ed.ac.uk/ Tracer 2014.

30. Lu L, Jia M, Ma Y, Yang L, Chen Z, Ho DD, Jiang Y, Zhang $L$. The changing face of HIV in China. Nature. 2008:455(7213):609-11. 
31. Mahy M, Autenrieth CS, Stanecki K, Wynd S. Increasing trends in HIV prevalence among people aged 50 years and older: evidence from estimates and survey data. AIDS. 2014;28(Suppl 4):S453-9.

32. Autenrieth CS, Beck EJ, Stelzle D, Mallouris C, Mahy M, Ghys P. Global and regional trends of people living with HIV aged 50 and over: estimates and projections for 2000-2020. PLoS One. 2018;13(11):e0207005.

33. Wei H, Xing H, Hsi JH, Jia M, Feng Y, Duan $S$, He C, Yao S, Ruan Y, He X, et al. The sexually driven epidemic in youths in China's southwestern border region was caused by dynamic emerging multiple recombinant HIV1 strains. Sci Rep. 2015;5:11323.

34. Chunlei L. EpidemiologicalCharacteristics ofHIV/AIDS in Yibin City, 20042013. J Occup Health Damage. 2015;5:275-77.

\section{Publisher's Note}

Springer Nature remains neutral with regard to jurisdictional claims in published maps and institutional affiliations.

Ready to submit your research? Choose BMC and benefit from:

- fast, convenient online submission

- thorough peer review by experienced researchers in your field

- rapid publication on acceptance

- support for research data, including large and complex data types

- gold Open Access which fosters wider collaboration and increased citations

- maximum visibility for your research: over $100 \mathrm{M}$ website views per year

At $B M C$, research is always in progress.

Learn more biomedcentral.com/submissions 